\title{
Ayvalık (Balıkesir/Turkey) Dune Plant Diversity, Threatening Factors and Solution Proposals
}

\author{
Fatih SATIL ${ }^{1}$, Gülendam TÜMEN ${ }^{1}$, Selami SELVİ ${ }^{2} *$
}

\begin{abstract}
Ayvalık and its environs are unique due to its coastal structure and natural beauties, and particularly the islands belonging to Ayvalık makes up the most important resource values. This study was carried out to determine the plant diversity in the coastal dunes of Ayvalık and its surroundings, to present the threat factors and to offer solutions. The study was conducted between 2016-2017. For this purpose; floristic and ecological observations were made in four seasons. As a result of floristic studies; 88 genera and 115 taxa belonging to 32 families were determined. Fabaceae (15 taxa), Asteraceae (14 taxa) and Amaranthaceae (8 taxa) families are dominant in the flora of the region. The most common taxa in dune areas are; Matthiola tricuspidata, Malcolmia flexuosa, Salicornia emericii, Cakile maritima, Anthemis rigida, A. tomentosa, Halimione portulacoides, Plantago coronopus subsp. commutata, Trifolium subterraneum, Eryngium maritimum, Allium sphaerocephalon and Euphorbia paralias. The role of human factors is particularly notable in a significant part of the coastal dunes in the region. Beach arrangements in dune areas, secondary housing construction in coastal areas, sand extraction from coasts, tourism, wetland drying and fires threaten dune habitats and plant species in the region. Especially due to the extensive use of the coastal areas and beach arrangements; The habitats of Sea daffodil (Pancratium maritimum) and Narcissus (Narcissus tazetta) have been greatly damaged. Moreover, the fact that $P$. maritimum bulbs were collected by the people there greatly reduced the populations of this species. It also is given solution proposals for protection of dune plants.
\end{abstract}

Keywords: Balıkesir, biodiversity, ecology, flora

\footnotetext{
${ }^{1}$ Fatih SATIL (Orcid ID: 0000-0002-4938-1161), Gülendam TÜMEN (Orcid ID: 0000-0002-7457-3341), Balıkesir University, Faculty of Science \& Arts, Department of Biology, Cagis Kampüs, Balıkesir, Turkey

2 Selami SELVİ (Orcid ID: 0000-0002-9959-6945), Balıkesir University, Altınoluk Vocational School, Department of Plant and Animal Plants, Edremit, Balıkesir, Turkey

*Sorumlu Yazar / Corresponding Author: Selami SELVI, e-mail: sselvi2000@yahoo.com 


\section{INTRODUCTION}

The coastal areas, which constitute only $4 \%$ of the earth, contain about $1 / 3$ of the world's population. The coasts are important transitional areas between water environments and terrestrial environments, represented by areas of different sizes. Coastal areas are defined as actual ecotons due to their own ecological characteristics (Wiedemann, 1984; Odum and Barrett; 2008; Avc1, 2017a,b). Coastal dunes are complex systems in terms of growing conditions of vegetation. Properties such as low amount of plant nutrients and organic matter in sand dunes, high permeability rate, direct sunlight exposure and high temperatures, highly effective wind, mobility of the surface and exposure to seawater with high salt content are some of the important factors limiting the development of vegetation. Turkey has a total of 105 coastal dunes in 23 out of 26 coastal cities and their total area is 3557 ha. The length of the coasts in our country is $8333 \mathrm{~km}, 2805 \mathrm{~km}$ of which is in the Aegean, $1695 \mathrm{~km}$ in the Black Sea, $1577 \mathrm{~km}$ in the Mediterranean Sea, $1189 \mathrm{~km}$ in the Marmara Region, in Çanakkale Strait and Istanbul Bosphorus and $1067 \mathrm{~km}$ on the islands (Uslu, 1988; Avc1 et al 2015). According to Erinç (2001); Turkey's coastal dunes are observed in 75 different areas. 8 of these coastal dunes are located in Balikesir province and three of these areas (Çıplak Island, Sarımsaklı and Altınova) are located in Ayvalık district (Table 1).

Table 1. Coastal sand dunes in Balıkesir (Erinç 2001).

\begin{tabular}{lll}
\hline No & District & Dune field name \\
\hline 1 & Ayvalık & Altınova \\
2 & Ayvalık & Sarımsaklı \\
3 & Ayvalık & Yumra cape (Badavut beach) \\
4 & Burhaniye & Armutova \\
5 & Edremit & Çoruk \\
6 & Erdek & Belkıs tombolo \\
7 & Gönen & Gönen delta \\
8 & Gönen & Karabiga Delta \\
\hline
\end{tabular}

Apart from the flora of the area between Edremit Bay-Gökova Gulf near the study field (Akyol and Gemici 2017a,b), the flora and vegetation of the surrounding area of KaraburunAkdağ (Bekat and Seçmen 1988) and vegetation of Foça, Aliağa and Çandarlı region (Bekat 1980), there is a study carried out regarding the dune flora of Ayvalik. The coasts are quite important ecosystems in that they are transitional regions (ecotone) where marine and terrestrial ecosystems intersect. Coastal ecosystems are essential in terms of biodiversity and the balance maintained between sea and land (Akgün and Türk, 2011). This study was carried out to determine the plant diversity in the coastal dunes of Ayvalık and its surroundings, and to offer solutions to the problems by presenting the factors threatening this wealth.

\section{MATERIALS AND METHODS}

The study was carried out in Yumra Cape, Sarmisaklı and Altınova coastal dunes located in Ayvalık (Figure 1). In this study, which was carried out between 2016-2017, the plant diversity and environmental problems were determined through the four-season floristic and ecological observations made in the field. In floristic studies, dune and salt plant species in the area were collected and made into herbarium materials. 


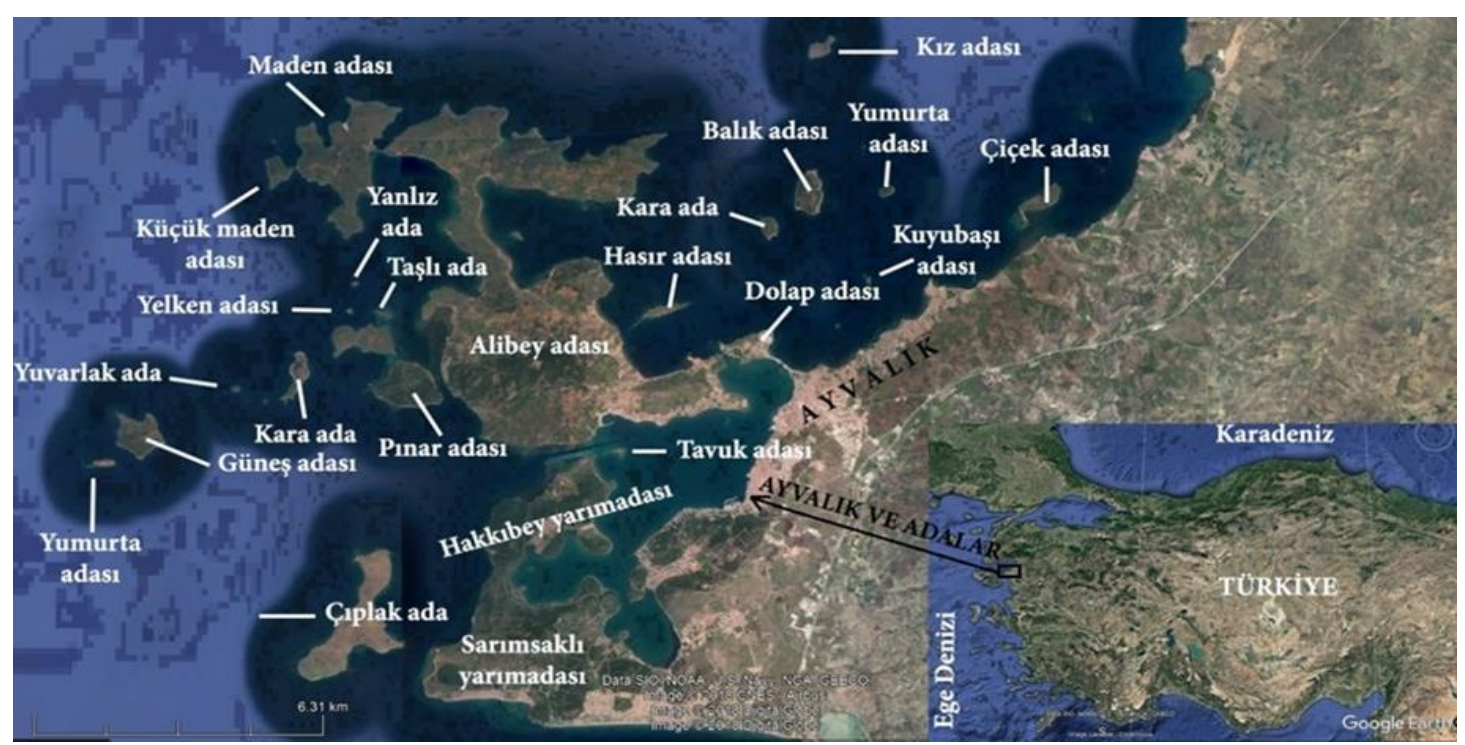

Figure 1. The map of study area.

Voucher specimens were deposited in the Botany Laboratory, Altınoluk Vocational School, Balıkesir University. The main source for the identification of the collected plants was mainly Flora of Turkey, Mountain Flora of Greece, Flora of Europae and floristic studies done in the region (Davis 1965-1985; Davis et al. 1988; Güner et al. 2000; Strid and Tan 1991; Tutin et al. 1976). Turkish names of identified plants are given according to Güner et al. (2012). In addition, the plant name authors are given according to Brummit and Powell (1992).

\section{RESULTS AND DISCUSSION}

In the field; 88 genera and 115 taxa belonging to 32 families were determined (Table 1).Fabaceae (15), Asteraceae (14) and Amaranthaceae ( 8) are the families with the most taxa (Figure 2). These three families account for about $32 \%$ of the taxa found in the area.

The most taxa-containing genus in the field area are Limonium (5), Allium (5) Anthemis (4) and Plantago (4) (Figure 3).

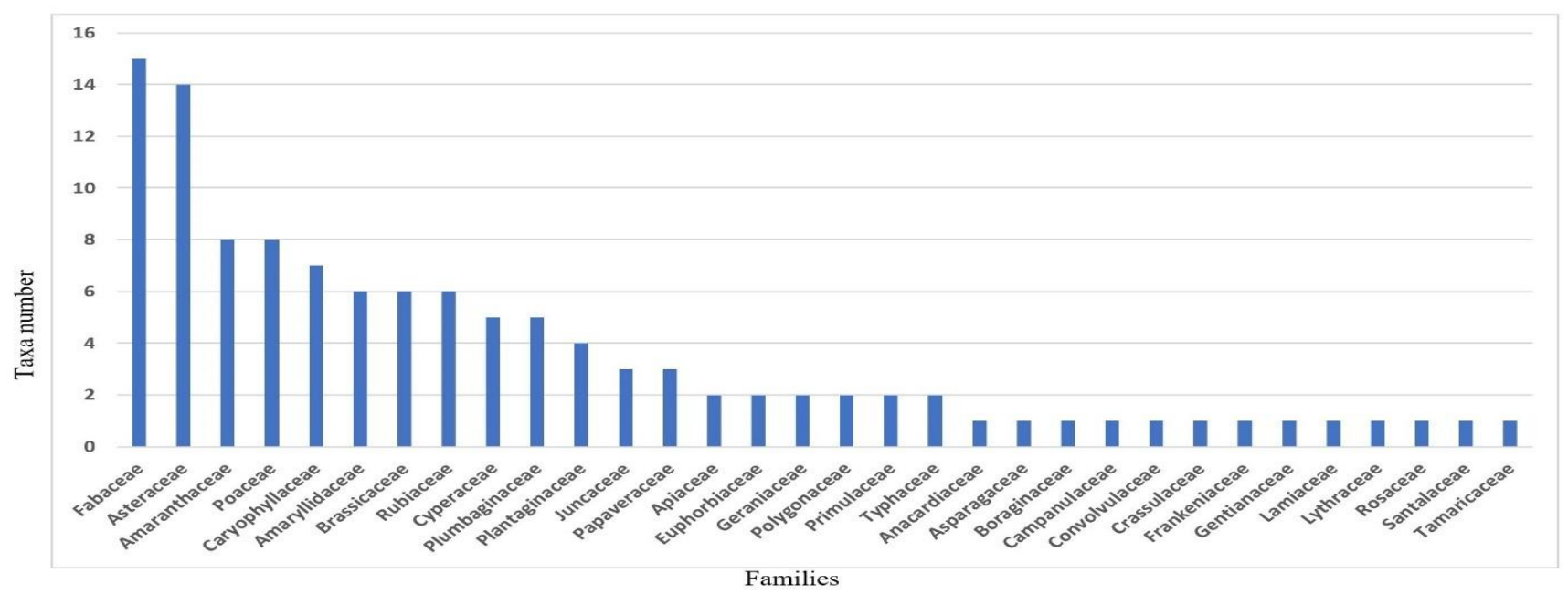

Figure 2. Families identified in the area and taxon numbers 


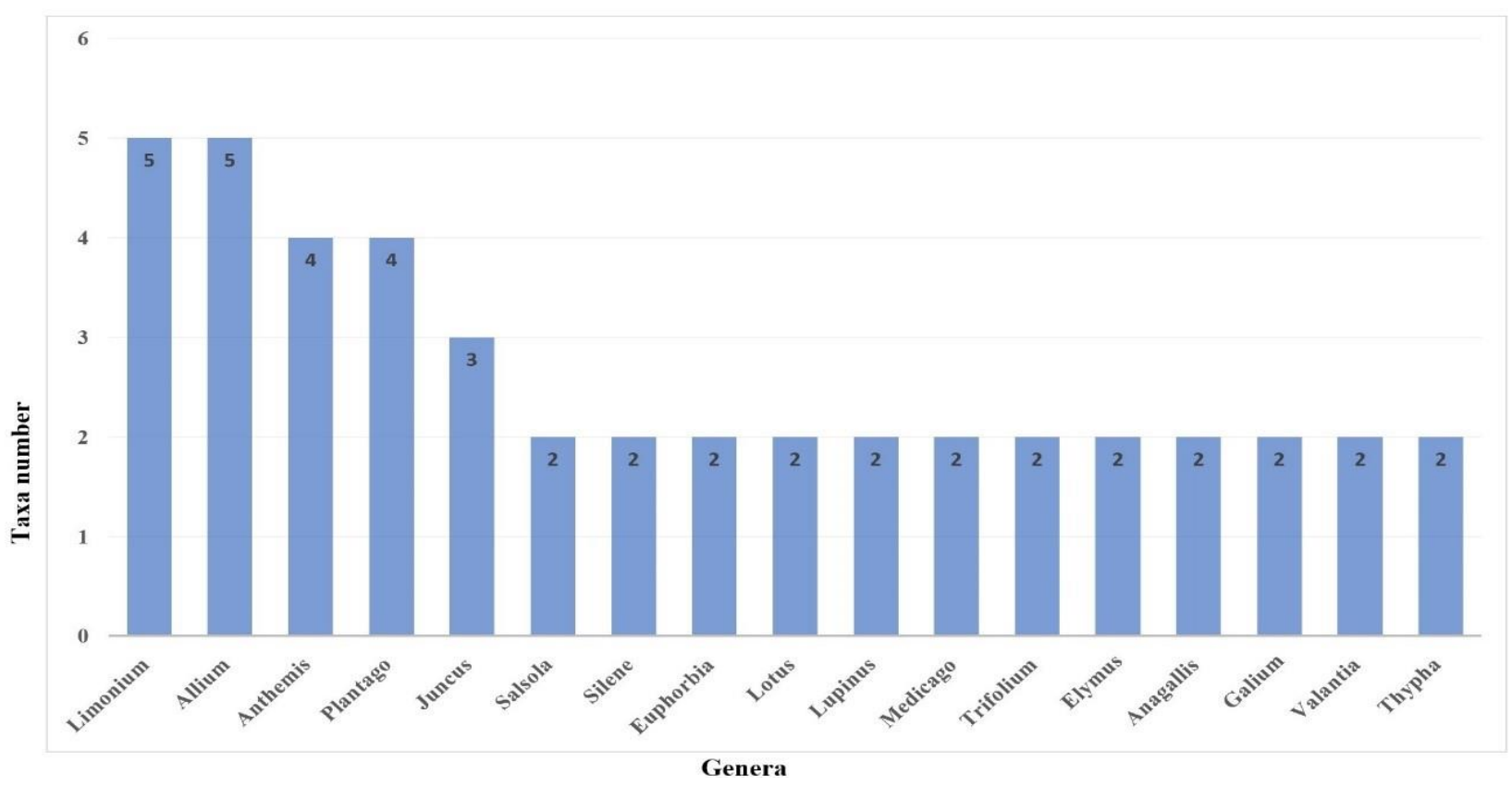

Figure 3. The most genera and taxa found in the region

Table 2. The flora of Ayvalik dunes.

\begin{tabular}{|c|c|c|c|c|}
\hline Family & Taxon & $\begin{array}{l}\text { Vernacular } \\
\text { Name }\end{array}$ & Locality and the voucher number & $\begin{array}{l}\text { Phytogeographical } \\
\text { Region }\end{array}$ \\
\hline Amaranthaceae & Amaranthus viridis $\mathrm{L}$. & Yeşilibik & $\begin{array}{l}\text { Southern of Sarımsak Peninsula, 07.05.2016 (SV } \\
\text { 1812) }\end{array}$ & - \\
\hline Amaranthaceae & Atriplex lasiantha Boiss. & Deliunluca & Alibey Island, Çatal Hill, 06.07.2017 (SV 2022) & - \\
\hline Amaranthaceae & Beta maritima L.var. maritima & Kıyıpancarı & $\begin{array}{l}\text { Small Maden Island, Sarımsak Peninsula, } \\
\text { 17.06.2016 (SV 1815) }\end{array}$ & - \\
\hline Amaranthaceae & $\begin{array}{l}\text { Halimione portulacoides }(\mathrm{L} .) \\
\text { Allen }\end{array}$ & Kocabetne & All coastline, 06.07.2017 (SV 2023) & - \\
\hline Amaranthaceae & Salicornia emericii Duval-Jouve & Denizbörülcesi & Sarımsak Peninsula, Tuzla, 06.07.2017 (SV 2025) & - \\
\hline Amaranthaceae & Salsola kali L. & Döngele & $\begin{array}{l}\text { Alibey Island, Patrice, Çatal Hill, 25.04.2016 (SV } \\
1768)\end{array}$ & - \\
\hline Amaranthaceae & S. soda $\mathrm{L}$. & Denizfasülyesi & Alibey Island, Balık Island, 15.04.2016 (SV 1745) & - \\
\hline Amaranthaceae & Suaeda prostrata Pall. & Yatıkcirim & $\begin{array}{l}\text { Alibey Island, Patrice, Çatal Hill, 15.04.2016 (SV } \\
\text { 1747) }\end{array}$ & - \\
\hline Amaryllidaceae & Allium ampeloprasum $\mathrm{L}$. & Pirasa & $\begin{array}{l}\text { Alibey Island, Hakkıbey Peninsula, 25.04.2016 } \\
\text { (SV 1765) }\end{array}$ & Mediterranean \\
\hline Amaryllidaceae & A. commutatum Guss. & İtdirseği & Kamıs Island, 15.04.2017, (SV 1946) & Mediterranean \\
\hline Amaryllidaceae & A. roseum L. subsp. roseum & Gülsoğanı & $\begin{array}{l}\text { Alibey Island, Alibey Hill, Hakkıbey Peninsula, } \\
\text { 15.04.2016 (SV 1740) }\end{array}$ & Mediterranean \\
\hline Amaryllidaceae & $\begin{array}{l}\text { A. sphaerocephalon L. subsp. } \\
\text { sphaerocephalon }\end{array}$ & Y1lan sarmısağ1 & $\begin{array}{l}\text { Alibey İsland Alibey Tepe, Hakkıbey Peninsula, } \\
\text { 15.04.2016 (SV 1738) }\end{array}$ & Euro-Sibirian \\
\hline Amaryllidaceae & Narcissus tazetta L. subsp. tazetta & Nergiz & Alibey Island, Duba, 07.05.2016 (SV 1817) & - \\
\hline Amaryllidaceae & Pancratium maritimum $\mathrm{L}$. & Kumzambağ & Alibey Island, Patrice, 07.05.2016 (SV 1821) & Mediterranean \\
\hline Anacardiaceae & Pistacia lentiscus L. & Sakızağacı & $\begin{array}{l}\text { Alibey Island, Çatal Hill, Pınar Island, 25.04.2016 } \\
\text { (SV 1769) }\end{array}$ & Mediterranean \\
\hline Apiaceae & Crithmum maritimum $\mathrm{L}$. & Denizteresi & $\begin{array}{l}\text { Southwest of Sarımsak Peninsula, 05.04.2016 (SV } \\
\text { 1722) }\end{array}$ & - \\
\hline Apiaceae & Eryngium maritimum L. & Kumboğadikeni & $\begin{array}{l}\text { Sarımsak Peninsula, Tuz cape, 05.04.2016 (SV } \\
\text { 1719) }\end{array}$ & - \\
\hline Asparagaceae & Drimia maritima (L.) Stearn & Kumörümcekotu & Pinar Island, 15.04.2017, (SV 1942) & - \\
\hline
\end{tabular}


Ayvalık (Balıkesir/Turkey) Dune Plant Diversity, Threatening Factors and Solution Proposals

\begin{tabular}{|c|c|c|c|c|}
\hline Asteraceae & $\begin{array}{l}\text { Achillea maritima (L.) Ehrend. \& } \\
\text { Y.P.Guo subsp. maritima }\end{array}$ & Çocukotu & $\begin{array}{l}\text { Eastern of Hakkıbey Peninsula, 05.04.2016 (SV } \\
\text { 1715) }\end{array}$ & - \\
\hline Asteraceae & Anthemis rigida Boiss. ex Heldr. & Diripapatya & $\begin{array}{l}\text { Alibey Island, Sarımsak Peninsula, 15.04.2016 } \\
\text { (SV 1736) }\end{array}$ & Mediterranean \\
\hline Asteraceae & A. tomentosa L. subsp. tomentosa & Sahilpapatyası & $\begin{array}{l}\text { Southern of Sarımsak Peninsula, 05.04.2016 (SV } \\
1710)\end{array}$ & Mediterranean \\
\hline Asteraceae & Bellis anпиа $\mathrm{L}$. & Çayır güzeli & Alibey Island, Patrice, 07.05.2016 (SV 1824) & Mediterranean \\
\hline Asteraceae & $\begin{array}{l}\text { Cardopatium corymbosum (L.) } \\
\text { Pers. }\end{array}$ & Kurtludiken & Alibey Island, Duba, 05.04.2016 (SV 1725) & Mediterranean \\
\hline Asteraceae & Carthamus caeruleus L. & Gökaspir & Alibey Island, Duba, 15.04.2016 (SV 1730) & Mediterranean \\
\hline Asteraceae & $\begin{array}{l}\text { Cladanthus mixtus (L.) Oberpr. \& } \\
\text { Vogt }\end{array}$ & Çirozpapatya & $\begin{array}{l}\text { Hakkıbey Peninsula, Cennet cape, 05.03.2016 (SV } \\
1610)\end{array}$ & Mediterranean \\
\hline Asteraceae & Cota altissima (L.) J.Gay & Köpek papatyası & Alibey Island, Duba, 25.04.2016 (SV 1772) & - \\
\hline Asteraceae & $\begin{array}{l}\text { Cota tinctoria }(\mathrm{L}) \mathrm{J} . \text { Gay var. } \\
\text { tinctoria }\end{array}$ & Boyacıpapatyası & $\begin{array}{l}\text { Alibey Island Patrice, Sarımsak Peninsula, } \\
\text { 15.04.2016 (SV 1734) }\end{array}$ & - \\
\hline Asteraceae & $\begin{array}{l}\text { Crepis commutata (Spreng.) } \\
\text { Greuter }\end{array}$ & Delikıskıs & Çıplak Island, 25.04.2016 (SV 1775) & - \\
\hline Asteraceae & $\begin{array}{l}\text { Hedypnois rhagadioloides }(\mathrm{L} .) \\
\text { Hayek subsp. cretica }\end{array}$ & Sünnetlice & $\begin{array}{l}\text { Alibey Island, Duba, Patrice, Çıplak Island, } \\
\text { 06.07.2017 (SV 2019) }\end{array}$ & Mediterranean \\
\hline Asteraceae & Helichrysum orientale (L.) DC. & Sarısolmaz & Alibey Island, Patrice, 07.05.2016 (SV 1826) & Mediterranean \\
\hline Asteraceae & $\begin{array}{l}\text { Helminthotheca echioides (L.) } \\
\text { Holub. }\end{array}$ & Billurdüğme & Alibey Island, Duba, 05.03.2016 (SV 1618) & - \\
\hline Asteraceae & Senecio vernalis Waldst. et Kit. & Kanaryaotu & $\begin{array}{l}\text { Maden Island, Hakkıbey Peninsula, 25.04.2016 } \\
\text { (SV 1781) }\end{array}$ & - \\
\hline Boraginaceae & Echium angustifolium Mill. & Engerek otu & $\begin{array}{l}\text { Sarımsak Peninsula, Şeytansofrası, 05.03.2016 (SV } \\
1605)\end{array}$ & Mediterranean \\
\hline Brassicaceae & Alyssum umbellatum Desv. & Şişkinkevke & $\begin{array}{l}\text { Badavut, Sarımsaklı Peninsula, 25.04.2016 (SV } \\
\text { 1777) }\end{array}$ & Mediterranean \\
\hline Brassicaceae & Cakile maritima Scop. & Kumteresi & $\begin{array}{l}\text { Alibey Island, Çatal Hill, Sarımsak Peninsula, } \\
\text { 05.03.2016 (SV 1603) }\end{array}$ & - \\
\hline Brassicaceae & Eruca vesicaria (L.) Cav. & Roka & Alibey Island, Alibey Hill, 05.03.2016 (SV 1607) & - \\
\hline Brassicaceae & $\begin{array}{l}\text { Malcolmia flexuosa (Sibth. \& } \\
\text { Sm.) Sibth. \& Sm. }\end{array}$ & Kayaşebboyu & $\begin{array}{l}\text { Hakkıbey Peninsula, Timarhane Hill, 29.03.2016 } \\
\text { (SV 1705) }\end{array}$ & Mediterranean \\
\hline Brassicaceae & Matthiola tricuspidata (L.) R. Br. & Öküzşebboyu & $\begin{array}{l}\text { Eastern of Alibey Island, Patrice second } \\
\text { bay,16.03.2017 (SV 1909) }\end{array}$ & Mediterranean \\
\hline Brassicaceae & Raphanus raphanistrum $\mathrm{L}$. & Yabani turp & $\begin{array}{l}\text { Alibey Island, Duba,Patrice,Sarımsak Peninsula, } \\
\text { 17.03.2016 (SV 1635) }\end{array}$ & - \\
\hline Campanulaceae & $\begin{array}{l}\text { Jasione montana L.subsp. } \\
\text { montana }\end{array}$ & Dağgökçesi & $\begin{array}{l}\text { Southern of Sarımsak Peninsula, 17.03.2016 (SV } \\
1637)\end{array}$ & - \\
\hline Caryophyllaceae & $\begin{array}{l}\text { Arenaria leptoclados (Reich.) } \\
\text { Guss. }\end{array}$ & Kum otu & Maden Island, 28.03.2017, (SV 1921) & - \\
\hline Caryophyllaceae & Paronychia echinulata Chater & Kıyıkepekotu & Pinar Island, 15.04.2017, (SV 1939) & Mediterranean \\
\hline Caryophyllaceae & Sagina maritima G.Don & Arsıarinotu & Kamış Island, 15.04.2017, (SV 1948) & - \\
\hline Caryophyllaceae & Silene behen $\mathrm{L}$. & Akkıvşak & $\begin{array}{l}\text { Alibey Island, Patrice, Kamış Island, 16.03.2017 } \\
\text { (SV 1912) }\end{array}$ & - \\
\hline Caryophyllaceae & Silene gallica $\mathrm{L}$. & Serçeçiçeği & $\begin{array}{l}\text { Alibey Island, Patrice Maden Island, 16.03.2017 } \\
\text { (SV 1914) }\end{array}$ & - \\
\hline Caryophyllaceae & Spergula arvensis $\mathrm{L}$. & Tarla kişnişi & Alibey Island, Patrice, 01.05.2016 (SV 1786) & - \\
\hline Caryophyllaceae & Spergularia marina (L.) Besser & Sahilremilotu & $\begin{array}{l}\text { Alibey Island, Küçük Maden Island, 01.05.2016 } \\
\text { (SV 1790) }\end{array}$ & - \\
\hline Convolvulaceae & Cressa cretica $\mathrm{L}$. & Reçineçiçeği & Sarımsak Peninsula, Tuzla, 15.06.2016, (SV 1845) & - \\
\hline Crassulaceae & $\begin{array}{l}\text { Sedum litoreum Guss. var. } \\
\text { litoreum }\end{array}$ & Kıyıkoruğu & $\begin{array}{l}\text { Alibey Island Patrice second bay, Maden Island, } \\
\text { 25.04.2016 (SV 1782) }\end{array}$ & Mediterranean \\
\hline Cyperaceae & $\begin{array}{l}\text { Bolboschoenus maritimus (L.) } \\
\text { Palla subsp. maritimus }\end{array}$ & Sandalyesazı & $\begin{array}{l}\text { Sarımsak Peninsula, Şeytansofrası, 29.03.2016 (SV } \\
\text { 1700) }\end{array}$ & - \\
\hline Cyperaceae & Carex muricata L.subsp. muricata & Çengelsazı & $\begin{array}{l}\text { Alibey Island Patrice Second bay, 01.05.2016 (SV } \\
\text { 1792) }\end{array}$ & Euro-Sibirian \\
\hline Cyperaceae & Cyperus capitatus Vandelli & Şehvetotu & $\begin{array}{l}\text { Southern of Sarımsak Peninsula, 29.03.2016 (SV } \\
\text { 1698) }\end{array}$ & - \\
\hline Cyperaceae & $\begin{array}{l}\text { Eleocharis palustris (L.) Roem. \& } \\
\text { Schult.subsp. palustris }\end{array}$ & Delisaz & Hakkıbey Peninsula, 29.03.2016 (SV 1695) & - \\
\hline
\end{tabular}


Ayvalık (Balıkesir/Turkey) Dune Plant Diversity, Threatening Factors and Solution Proposals

\begin{tabular}{|c|c|c|c|c|}
\hline Cyperaceae & $\begin{array}{l}\text { Scirpoides holoschoenus (L.) } \\
\text { Soják subsp. holoschoenus }\end{array}$ & Vurla & $\begin{array}{l}\text { Eastern of Alibey Island, Sarımsaklı beach, } \\
01.05 .2016 \text { (SV 1788) }\end{array}$ & - \\
\hline Euphorbiaceae & Euphorbia paralias L. & Kumsütleğeni & $\begin{array}{l}\text { Alibey Island, Çatal Hill, Pınar Island, 29.03.2016 } \\
\text { (SV 1690) }\end{array}$ & Mediterranean \\
\hline Euphorbiaceae & Euphorbia peplis L. & Kıyısütleğeni & $\begin{array}{l}\text { Alibey Island, Çatal Hill, Sarımsak Peninsula, } \\
\text { 01.05.2016 (SV 1794) }\end{array}$ & $c_{1}$ \\
\hline Fabaceae & $\begin{array}{l}\text { Hippocrepis unisiliquosa } \mathrm{L} . \\
\text { subsp. unisiliquosa }\end{array}$ & Atnalı & Alibey Island, Sivrice cape, 09.07.2016, (SV 1855) & - \\
\hline Fabaceae & $\begin{array}{l}\text { Hymenocarpos circinnatus (L.) } \\
\text { Savi }\end{array}$ & Pulluot & $\begin{array}{l}\text { Alibey Island, Sivrice cape, Pinar Island, } \\
\text { 09.07.2016, (SV 1851) }\end{array}$ & Mediterranean \\
\hline Fabaceae & Lotus ornithopodioides L. & Civcivayağg & $\begin{array}{l}\text { Southern of Sarımsak Peninsula, 29.03.2016 (SV } \\
1683 \text { ) }\end{array}$ & - \\
\hline Fabaceae & $\begin{array}{l}\text { L. corniculatus L. var. } \\
\text { corniculatus }\end{array}$ & Gazalboynuzu & $\begin{array}{l}\text { Hakkıbey Peninsula, Tımarhane Hill, 29.03.2016 } \\
\text { (SV 1685) }\end{array}$ & - \\
\hline Fabaceae & $\begin{array}{l}\text { Lupinus angustifolius L. subsp. } \\
\text { angustifolius }\end{array}$ & Acıbakla & $\begin{array}{l}\text { Alibey Island, Duba, Hakkıbey Peninsula, } \\
\text { 17.03.2016 (SV 1680) }\end{array}$ & - \\
\hline Fabaceae & $\begin{array}{l}\text { L. angustifolius (Desv.) } \\
\text { Cout.subsp. reticulatus }\end{array}$ & Yahudibaklası & Badavut, 01.05.2016 (SV 1801) & Mediterranean \\
\hline Fabaceae & Medicago marina $\mathrm{L}$. & Sahil yoncası & $\begin{array}{l}\text { Southern of Sarımsak Peninsula, 17.03.2016 (SV } \\
1638)\end{array}$ & - \\
\hline Fabaceae & Medicago orbicularis (L.) Bart. & Paralık & $\begin{array}{l}\text { Hakkıbey Peninsula, Timarhane Hil, 01.03.2016 } \\
\text { (SV 1600) }\end{array}$ & - \\
\hline Fabaceae & Onobrychis caput-galli (L.) Lam. & Pitrakkorunga & $\begin{array}{l}\text { Alibey Island Alibey Hill, Çıplak Island, } \\
\text { 05.03.2016 (SV 1602) }\end{array}$ & Mediterranean \\
\hline Fabaceae & Ornithopus compressus L. & Kuşayağ1 & $\begin{array}{l}\text { Alibey Island, Duba, Sarımsak Peninsula, } \\
\text { 05.03.2016 (SV 1598) }\end{array}$ & Mediterranean \\
\hline Fabaceae & $\begin{array}{l}\text { Scorpiurus muricatus L. var. } \\
\text { subvillosus (L.) Fiori }\end{array}$ & Akrep kuyruğu & $\begin{array}{l}\text { Alibey Island, Patrice, Çatal Hill, 09.07.2016, (SV } \\
\text { 1858) }\end{array}$ & Mediterranean \\
\hline Fabaceae & Trifolium repens $\mathrm{L}$. var. repens & Aküçgül & $\begin{array}{l}\text { Southern of Sarımsak Peninsula, 17.03.2016 (SV } \\
1640)\end{array}$ & - \\
\hline Fabaceae & Trifolium subterraneum $\mathrm{L}$. & Yeraltıüç̧ülü & Alibey Island, Patrice, 17.03.2016 (SV 1645) & - \\
\hline Fabaceae & $\begin{array}{l}\text { Trigonella coerulescens } \\
\text { (M.Bieb.) Halácsy subsp. } \\
\text { ayvalikensis Erdoğan Selvi\& } \\
\text { Tümen (ENDEMIC) }\end{array}$ & Ayvalık çemeni & $\begin{array}{l}\text { Alibey Island, Duba, Sarımsak Peninsula, } \\
\text { 17.03.2016 (SV 1667) }\end{array}$ & - \\
\hline Fabaceae & $\begin{array}{l}\text { Vicia villosa Roth subsp. } \\
\text { eriocarpa (Hausskn.) P.W.Ball. }\end{array}$ & Boğala & Alibey Island, Patrice, 17.03.2016 (SV 1669) & - \\
\hline Frankeniaceae & Frankenia hirsuta $\mathrm{L}$. & Tülpembe & $\begin{array}{l}\text { Alibey Island Alibey Hill, Sarımsak Peninsula, } \\
\text { 17.03.2016 (SV 1665) }\end{array}$ & - \\
\hline Gentianaceae & $\begin{array}{l}\text { Centaurium maritimum (L.) } \\
\text { Fritsch }\end{array}$ & Sarıgelindüğmesi & Alibey Island, Duba, 09.07.2016, (SV 1860) & Mediterranean \\
\hline Geraniaceae & $\begin{array}{l}\text { Erodium acaule (L.) Becherer et } \\
\text { Thell. }\end{array}$ & Leylekgagası & Alibey Island, Duba, 25.04.2017 (SV 1955) & - \\
\hline Geraniaceae & Geranium molle $\mathrm{L}$ & Yumuşak 1tır & $\begin{array}{l}\text { Southern of Sarımsak Peninsula, 21.06.2016 (SV } \\
1855)\end{array}$ & - \\
\hline Juncaceae & Juncus acutus L. & Kofa & Alibey Island, Çatal Hill, 15.07.2016 (SV 1870) & - \\
\hline Juncaceae & J. capitatus Weigel & Topak kofa & Hakkıbey Peninsula, 17.03.2016 (SV 1660) & - \\
\hline Juncaceae & J. gerardi Loisel. subsp. gerardi & Erkek kofa & Alibey Island, Duba, 09.07.2016, (SV 1862) & - \\
\hline Lamiaceae & Vitex agnus-castus $\mathrm{L}$. & Hayıt & $\begin{array}{l}\text { Sarımsak Peninsula, Tuzla lake, 15.06.2016, (SV } \\
1835)\end{array}$ & Mediterranean \\
\hline Lythraceae & $\begin{array}{l}\text { Lythrum tribracteatum Salzm. ex } \\
\text { Ten. }\end{array}$ & Üçaklarotu & Hakkıbey Peninsula, 17.03.2016 (SV 1658) & - \\
\hline Papaveraceae & Glaucium flavum Crantz & Gündürmelalesi & Sarımsak Peninsula, Tuzla, 15.06.2016, (SV 1842) & - \\
\hline Papaveraceae & $\begin{array}{l}\text { Hypecoum procumbens subsp. } \\
\text { atropunctatum Å.E.Dahl }\end{array}$ & Düğmecik & $\begin{array}{l}\text { Southern of Sarımsak Peninsula, 21.06.2016 (SV } \\
1854)\end{array}$ & Mediterranean \\
\hline Papaveraceae & Papaver rhoeas L. var. rhoeas & Gelincik & $\begin{array}{l}\text { Alibey Island, Hakkıbey Peninsula, 15.07.2016 } \\
\text { (SV 1866) }\end{array}$ & - \\
\hline Plantaginaceae & Plantago bellardii All. & Babadeşen & Alibey Island, Patrice, 17.03.2016 (SV 1649) & Mediterranean \\
\hline Plantaginaceae & $\begin{array}{l}\text { Plantago coronopus L. subsp. } \\
\text { commutata (Guss.) Pilger }\end{array}$ & Çı̆gnak & $\begin{array}{l}\text { Alibey Island, Küçük Maden Island, 25.04.2017 } \\
\text { (SV 1958) }\end{array}$ & Mediterranean \\
\hline Plantaginaceae & Plantago lagopus L. & Kurkdamarotu & $\begin{array}{l}\text { Alibey Island,Sarımsak Peninsula, 25.04.2017 (SV } \\
\text { 1965) }\end{array}$ & Mediterranean \\
\hline
\end{tabular}


Ayvalık (Balıkesir/Turkey) Dune Plant Diversity, Threatening Factors and Solution Proposals

\begin{tabular}{|c|c|c|c|c|}
\hline Plantaginaceae & Plantago lanceolata $\mathrm{L}$. & Damarlica & $\begin{array}{l}\text { Sarımsak Peninsula, Pınar Island, 15.06.2016, (SV } \\
1836)\end{array}$ & - \\
\hline Plumbaginaceae & Limonium sinuatum (L.) Mill. & Denizlavantası & $\begin{array}{l}\text { Alibey Island, Maden Island, 15.07.2016 (SV } \\
\text { 1868) }\end{array}$ & Mediterranean \\
\hline Plumbaginaceae & L. angustifolium (Tausch) Turrill & Sahilkaranfili & Alibey Island, Patrice, 25.04.2017 (SV 1966) & - \\
\hline Plumbaginaceae & L. bellidifolium (Gouan) Dumort & Hoşkuduzotu & Sarımsak Peninsula, Tuzla, 15.06.2016, (SV 1838) & Euro-Sibirian \\
\hline Plumbaginaceae & $\begin{array}{l}\text { L. graecum (Poir.) Rech.f. var. } \\
\text { graecum }\end{array}$ & Kumkaranfili & Alibey Island, Çatal Hill, 25.04.2017 (SV 1969) & Mediterranean \\
\hline Plumbaginaceae & L. virgatum (Willd.) Fourr. & C1lizot & Sarımsak Peninsula, 21.06.2016 (SV 1851) & Mediterranean \\
\hline Poaceae & $\begin{array}{l}\text { Brachypodium distachyon (L.) } \\
\text { P.Beauv. }\end{array}$ & Tekkılcan & $\begin{array}{l}\text { Alibey Island, Duba, Pınar Island, 09.05.2017 (SV } \\
\text { 1972) }\end{array}$ & Mediterranean \\
\hline Poaceae & $\begin{array}{l}\text { Echinochloa crus-galli (L.) P. } \\
\text { Beauv. }\end{array}$ & Darican & $\begin{array}{l}\text { Southern of Sarımsak Peninsula, 21.06.2016 (SV } \\
1853)\end{array}$ & - \\
\hline Poaceae & $\begin{array}{l}\text { Elymus farctus (Viv.) Runemark } \\
\text { ex Melderis }\end{array}$ & Cicora & East of Hakkıbey Peninsula, 17.03.2016 (SV 1650) & - \\
\hline Poaceae & $\begin{array}{l}\text { E. flaccidifolius (Boiss. \& Heldr.) } \\
\text { Melderis }\end{array}$ & Bataklıkcicorası & East of Hakkıbey Peninsula, 01.05.2016 (SV 1799) & Mediterranean \\
\hline Poaceae & $\begin{array}{l}\text { Holcus annuus Salzm. ex } \\
\text { C.A.Meyer }\end{array}$ & Kadife otu & Kamış Island, 29.06.2017 (SV 2011) & Mediterranean \\
\hline Poaceae & $\begin{array}{l}\text { Phragmites australis (Cav.) Trin. } \\
\text { ex Steudel }\end{array}$ & Kamış & $\begin{array}{l}\text { Alibey Island Duba, Sarımsak Peninsula, } \\
\text { 06.07.2017 (SV 2018) }\end{array}$ & Euro-Sibirian \\
\hline Poaceae & Poa bulbosa $\mathrm{L}$. & Yumrulusalkım & Alibey Island, Alibey Hill, 09.05.2017 (SV 1974) & - \\
\hline Poaceae & Stipa capensis Thunb. & Kumkılaçı & Maden Island, 28.03.2017, (SV 1927) & Mediterranean \\
\hline Polygonaceae & Polygonum maritimum $\mathrm{L}$. & Sicimlik & $\begin{array}{l}\text { Southern of Sarımsak Peninsula, Alibey Island, } \\
\text { 11.05.2016 (SV 1828) }\end{array}$ & - \\
\hline Polygonaceae & Rumex pulcher L. subsp. pulcher & Ekşilik & $\begin{array}{l}\text { Southern of Sarımsak Peninsula, Alibey Island, } \\
\text { 11.05.2016 (SV 1829) }\end{array}$ & - \\
\hline Primulaceae & $\begin{array}{l}\text { Anagallis arvensis L. var. } \\
\text { arvensis }\end{array}$ & Farekulağı & $\begin{array}{l}\text { Southern of Sarımsak Peninsula Alibey Island, } \\
\text { 11.05.2016 (SV 1832) }\end{array}$ & - \\
\hline Primulaceae & $\begin{array}{l}\text { A. arvensis L. var. parviflora } \\
\text { (Hoffmanns. \& Link) Ces. }\end{array}$ & Farekulağ 1 & Alibey Island, Patrice, 29.06.2017 (SV 2004) & Mediterranean \\
\hline Rosaceae & $\begin{array}{l}\text { Sarcopoterium spinosum }(\mathrm{L} .) \\
\text { Spach }\end{array}$ & Abdestbozan & $\begin{array}{l}\text { Alibey Island, Maden Island, Pınar Island, } \\
\text { 09.05.2017 (SV 1976) }\end{array}$ & - \\
\hline Rubiaceae & Crucianella imbricata Boiss. & Kurakhaçotu & $\begin{array}{l}\text { Southwest of Sarımsak Peninsula, 12.06.2017 (SV } \\
\text { 1997) }\end{array}$ & Mediterranean \\
\hline Rubiaceae & Galium aparine $\mathrm{L}$. & Çobansüzgeci & Kamış Island, 29.06.2017 (SV 2002) & - \\
\hline Rubiaceae & $\begin{array}{l}\text { G. brevifolium Sm. subsp. } \\
\text { insulare Ehrend. \& Schrönb.- } \\
\text { Tem. }\end{array}$ & Sünnetotu & Yumurta Island, 29.06.2017 (SV 2007) & Mediterranean \\
\hline Rubiaceae & Sherardia arvensis $\mathrm{L}$. & Gökörenotu & $\begin{array}{l}\text { Alibey Island, Patrice,Sarımsak Peninsula, } \\
\text { 09.05.2017 (SV 1979) }\end{array}$ & Mediterranean \\
\hline Rubiaceae & Valantia hispida $\mathrm{L}$. & Killı̈renotu & $\begin{array}{l}\text { Southern of Sarımsak Peninsula, Alibey Island, } \\
\text { 29.06.2017 (SV 1999) }\end{array}$ & Mediterranean \\
\hline Rubiaceae & Valantia muralis L. & Örenotu & Çıplak Island, 29.06.2017 (SV 2001) & Mediterranean \\
\hline Santalaceae & Thesium humile Vahl. & Bodurgüvelek & Alibey Island, Patrice, 09.05.2017 (SV 1982) & Mediterranean \\
\hline Tamaricaceae & Tamarix tetrandra Pall. ex Bieb. & Gezik & Alibey Island, Çatal Hill, 09.05.2017 (SV 1988) & - \\
\hline Typhaceae & Typha angustifolia $\mathrm{L}$. & Saz & $\begin{array}{l}\text { Southwest of Sarımsak Peninsula, 12.06.2017 (SV } \\
\text { 1992) }\end{array}$ & - \\
\hline Typhaceae & Typha domingensis Pers. & Şeytanmumu & $\begin{array}{l}\text { Southern of Sarımsak Peninsula, 12.06.2017 (SV } \\
\text { 1994) }\end{array}$ & - \\
\hline
\end{tabular}

Distribution of taxa to phytogeographic regions is as follows; The Mediterranean element is $39 \%$, the Euro-Siberian Element is $3 \%$, and the unknown is $58 \%$. In the study region, Iran-Turanian elements weren't found. According to IUCN, two taxa were identified as threatened categories. Local endemic Trigonella coerulescens subsp. ayvalikensis was evaluated as VU (Vulnerable) while Pancratium maritimum was evaluated in LC (Least Concern) category. 
The characteristic flora of the sand dunes in the region is dominated by Brassicaceae, Plantaginaceae and Amaranthaceae families. Cakile maritima, Matthiola tricuspidata and Malcolmia flexuosa from Brassicaceae family; Plantago coronopus subsp. commutata from Plantaginaceae family and Halimione portulacoides, Salsola kali and S. soda from Amaranthaceae family have been observed intensively along the coasts of Ayvalik.

The coastal dunes in the area pass from the sea shore line, after a width of 5-10 m towards the front dune, to the rear dune zone. In the predunes, one-year-old ephemeral plants are mainly observed and perennial and woody species are frequently found towards the rear dunes. In the front sand dunes in the area, Matthiola tricuspidata, Malcolmia flexuosa, Salicornia emericii, Cakile maritima, Anthemis rigida, A. tomentosa, Halimione portulacoides, Plantago coronopus, Lotus cytisoides, Trifolium subterraneum, Eryngium maritimum, Jasione montana, Allium sphaerocephalon, Euphorbia paralias, Trigonella coerulescens subsp. ayvalikensis and Limonium bellidifolium taxa were found intensively. However, in the sand dunes of Cunda, Matthiola tricuspidata, Malcolmia flexuosa, Anthemis rigida, A. tomentosa, Plantago coronopus, Lotus cytisoides, Trifolium subterraneum, Eryngium maritimum, Cakile maritima and Euphorbia paralias have been extensively distributed. In the sand dunes of Badavut, Halimione portulacoides, Jasione montana, Allium sphaerocephalon, Euphorbia paralias taxa are dense. In the sandy dunes of Sarımsakl1, Lotus cytisoides, Anthemis rigida, A. tomentosa taxa along with one-year endemic $T$. coerulescens subsp. ayvalikensis species are extensively distributed. Halimione portulacoides, Salicornia europaea and Limonium bellidifolium have densely formed sand meadows in the Altınova dunes. As we move towards the interior, there is a change in the type in that species such as
Sarcopoterium spinosum, Lavandula stoechas subsp. stoechas, Pistacia lentiscus, Limonium angustifolium, L. bellidifolium, L. graecum var. graecum, L. sinuatum, L. virgatum, Eryngium maritimum, Cardopatium corymbosum, Elymus farctus, Halimione portulacoides, Asphodelus aestivus and Inula viscosa have been distributed in the region. In the Altınova dunes, however, $L$. bellidifolium is in dense populations. In the inner part of the settled dune meadows, the $S$. spinosum and the A. aestivus are observed. In the environments similar to wetland and reed field on the shore, species demanding high level of watering such as Juncus acutus, J. capitatus, J. gerardi subsp. gerardi, and Phragmites australis are abundant. The only endemic taxa distributed in the sand dunes of Ayvalik is T. coerulescens subsp. ayvalikensis (Erdoğan et al. 2017). Furthermore, although Carthamus caeruleus are available in western countires, the only place in Turkey, where it is grown, is on the coasts of 3 localities of Cunda Island (Alpınar, 1997).

In the study, as a result of floristic and ecological observations, anthropogenic factors that threaten dune plant diversity and distribution areas were determined: In this study, Avc1 (2017a) pointed out that various human effects such as agricultural activities, urbanization and tourism have changed the coastal ecosystems and the most important factors causing damaging change in coastal ecosystems are anthropogenic factors. Similar anthropogenic effects were also heavily observed in the coastal dunes of Ayvalik. In particular, due to tourism and secondary housing construction in the region, changes in land use are important factors leading to habitat losses.

Distribution area of one-year endemic $T$. coerulescens subsp. ayvalikensis species (Ayvalık çemeni) is a beach area in the sand dunes of Sarımsaklı, Ayvalık. However, the fact that the flowering period of the species is long before the holiday season has prevented the species from being endangered. Moreover, it 
would be beneficial to protect the spreading area of the species against the beach arrangement and the grazing. In particular, it is damaging the flora of dune areas due to activities such as beach arrangement. Therefore, the habitats of $P$. maritimum and $N$. tazetta have been damaged and their populations on the island have been reduced. In addition, that the bulbs of sea daffodil is collected by the public has brought this species to the stage of extinction. Sand dunes with the habitats of species such as "sea daffodil" and "Narcissus" should be put under protection. From the habitats determined in the region, especially the areas located in the coastal part such as the coastal dunes, degraded coastal forests, beaches, wetlands and reed-straw areas, which are under pressure of heavy land use, should be primarily protected. Uncontrollability and ignorance are the basis of ecological problems in the region. Therefore, besides the biological, cultural and geomorphological resource values that are in the foreground in the ecological planning process, it is also important to raise awareness of the people in the region. In order to protect the landscaping, ecological, cultural and archaeological values of the Natural Park, restriction in human use of some areas in the region is required. For this purpose, regions with different levels of protection and use as well as sensitive ecosystems and values should be established in the Natural Park.

\section{CONCLUSION}

The species and evaluations identified in this study are thought to constitute the basis for the coastal management studies to be conducted in the future. The nature walks that will be done in Natural Park and introduce the plants and natural life of the environment will increase the interest in the matter. To that end, ecotourism routes should be determined in the area. On these trips which may be done on appropriate dates, the photos of the plants may be taken but their collection should never be allowed.
According to the (f) paragraph of article 9. of Environmental Law numbered 2872, "In order to ensure the sustainability of biodiversity, the protection of endangered and endangered species and rare plant and animal species is essential and prohibited to be traded in contradiction with the legislation". Those who destroy biodiversity in contravention of the provisions the $(\mathrm{k})$ paragraph of of article 20 of the same law are fined.

\section{REFERENCES}

Akgün A, Türk N, 2011. Mapping erosion susceptibility by a multivariate statistical method: A case study from the Ayvalık region, NW Turkey. Computers and Geosciences, 37: 1515-1524.

Akyol Y, Gemici Y, 2017a. Vegetation Ecology and Ecological Management of Biodiversity of Coastal Aegean (Between Edremit and Gökova Gulfs) in Turkey. Kastamonu Üniversitesi Orman Fakültesi Dergisi, 17(1): 116-123.

Akyol Y, Gemici Y, 2017b. Ege Kiy1ları (Edremit Körfezi-Gökova Körfezi Arası)'nın Florası. Nevşehir Bilim ve Teknoloji Dergisi, 6(2): 494- 517.

Alpınar K, 1997. Türkiye'de Carduncellus caeruleus (L.) C.Presl var. incisus DC.'un varlığı. OT Sistematik Botanik Dergisi, 4 (1): 43-48.

Avcı M, 2017a. Türkiye'nin Kıyı Kumulları, Bitki Örtüsü ve Önemi. International Symposium on Geomorphology. 12-14 October 2017, Elazığ/Türkiye, p. 114-121.

Avcı M, 2017b. Türkiye'nin Kıyı Kumullarında Bitki Örtüsü, Yasal ve Bilimsel Boyutlarıyla: Kıyı (Ed. H. Turoğlu ve H. Yiğitbaşığlu), Jeomorfoloji Derneği Yayını No: 1, Anka Matbaa, İstanbul, 63-92.

Avcı M, Avc1 S, Akkurt S, 2015. Coastal dune vegetation in Turkey: a geographical perspective. International Conference on the Mediterranean Coastal Environment Medcoast 2015, Varna, Bulgaria, 6-10 October 2015, vol.1: 397-405.

Bekat L, 1980. Karaburun-Akdağ çevresinin flora ve vejetasyonu. Ege Üniversitesi Fen Bilimleri Enstitüsü, Yüksek Lisans tezi, 34 s. İzmir. 
Bekat L, Seçmen Ö, 1988. Vegatation In der umgebung von Foça, Aliağa und Çandarl1, Journal of Faculty of Science Ege University Series B, 10 (2):15-27.

Brummitt RK, Powell CE (eds.), (1992). Authors of plant names, Royal Botanic Gardens, Kew, London.

Davis PH, 1965-1985. Flora of Turkey and the East Aegean Islands", Edinburgh Univ. Press, Vol. 1-9, 724s, Edinburgh,

Davis PH, Mill RR, Tan K, 1988. Flora of Turkey and the East Aegean Islands (Supplement). Edinburgh Univ. Press, 590s, Edinburgh, 10.

Erdoğan E, Selvi S, Tümen G, 2017. Trigonella coerulescens subsp. ayvalikensis (Fabaceae), a new taxon from Balikesir, Western Anatolia, Phytotaxa 319(2): 167-174.

Erinç S, 2001. Jeomorfoloji II (3.Basım) (Güncelleştirenler: A.Ertek ve C. Güneysu). Der Yay. No: 294, İstanbul.

Güner A, Özhatay N, Ekim T, Başer KHC, 2000. Flora of Turkey and East Aegean Islands 11.vol (suppl.2). Edinburgh Univ. Press, 656s, Edinburgh.
Güner A, Aslan S, Ekim, T, Vural M, Babaç, M.T. (edlr.), 2012. Türkiye Bitkileri Listesi (Damarlı Bitkiler), Nezahat Gökyiğit Botanik Bahçesi ve Flora Araştırmaları Derneği Yayını. İstanbul.

Odum EP, Barrett GW, 2008. Ekolojinin Temel İlkeleri (Çev. Ed. K. Işık). Ankara: Palme Yayıncılik.

Strid, A, Kit Tan, (eds.), 1991. Mountainflora of Greece Vol. 2., Edinburgh University Press. Edinburgh, UK.

Tutin, G., Heywood, VH., Burgers, NA., Moore, DM., Vlentine, DH, Walters, SM., Webb, DA., 1976. Flora Europaea. Cambridge Univ. Press, Cambridge.

Uslu T, 1988. Türkiye Kıyı Kumulları. IX. Ulusal Biyoloji Kongresi, 21-23 Eylül 1988, Sivas. 175- 183.

Wiedemann AM, 1984. The Ecology of Pasific Northwest Coastal Sand Dunes: A Community Profile, FWS/OBS 84/04. 